\title{
QUANTUM INVARIANTS GIVEN BY EVALUATION OF KNOT POLYNOMIALS ${ }^{1}$
}

\author{
H. R. MORTON \\ Department of Pure Mathematics, University of Liverpool, \\ PO Box 147, Liverpool, L69 3BX
}

\begin{abstract}
It is shown that the knot invariant arising from an irreducible representation of a quantum group is, under certain conditions, an evaluation of the Homfly or Dubrovnik polynomial of the knot.

Besides the known cases of the fundamental representation for each of the quantum groups in the series $A_{n}, B_{n}, C_{n}$ and $D_{n}$, the results cover the special cases of the 3-dimensional representation of $S U(2)$ and the 6-dimensional representation of $S U(4)$, which can be viewed as the fundamental representations of $S O(3)$ and $S O(6)$ respectively. The second of these cases leads to a new relation between an evaluation of the Dubrovnik polynomial of a knot and an evaluation of the Homfly polynomials of two 2-cables about the knot.
\end{abstract}

Keywords: Quantum group, Homfly polynomial, Dubrovnik polynomial, $S U(4)$, unitary invariants.

\section{Introduction}

In this paper I show how, under certain conditions, the knot invariant arising from an irreducible representation of a quantum group can readily be seen to be an evaluation of the Homfly or Dubrovnik polynomial of the knot.

It is known [11] that all the quantum invariants of a framed link $L$ for the quantum groups of type $A_{n}, B_{n}, C_{n}$ and $D_{n}$ can be calculated in terms of knot polynomials by using suitable linear combinations of the polynomials of satellites of $L$. I am concerned here with quantum invariants which can be given by one of the polynomials of $L$ itself. Besides the known cases of the fundamental representation for each of the quantum groups listed, the results cover the special cases of the 3-dimensional representation of $S U(2)$, which may also be viewed as the fundamental representation of $S O(3)$, and the 6-dimensional representation of $S U(4)$, which corresponds to the fundamental representation of $S O(6)$. These appear in theorem 3.

To prove the results in this paper it is only necessary to check some simple features of the representations; it should also be possible to derive the connections from an explicit identification of the quantum groups.

From the calculation of the quantum invariant in terms of polynomials of satellites in the case of $S U(4)$ theorem 4 gives a previously unknown relation between an evaluation of the Dubrovnik polynomial of a knot and of the Homfly polynomials of two 2-cables about the knot.

\footnotetext{
${ }^{1}$ Appears in the Journal of Knot Theory and its Ramifications, vol 2 (1993), 195-209.
} 


\section{The Homfly and Dubrovnik polynomials}

In what follows I shall make considerable use of link diagrams in representing both links and framed links. From a geometric point of view, a link is a collection of closed curves in $\mathbf{R}^{3}$ while a framed link is a link together with a choice of a neighbouring parallel curve for each component of the link. The diagrammatic study of both of these is readily handled by use of Reidemeister's three types of move, shown below.

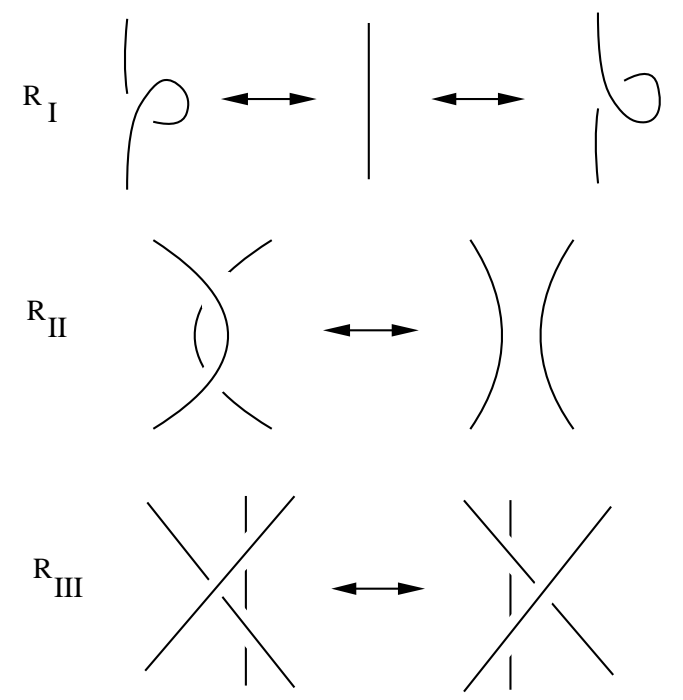

Links can be represented by link diagrams, up to a sequence of moves of types $R_{I}, R_{I I}$ and $R_{I I I}$, while a framed link can be represented by a link diagram up to moves of type $R_{I I}$ and $R_{I I I}$ only. In this second case the link diagram determines a 'diagrammatic' parallel to each component, by keeping just to one side of that component in the diagram, and thus determines a framed link, which is clearly unaltered by the moves $R_{I I}$ and $R_{I I I}$. It is possible to adjust the diagram of a link, by insertion of curls using move $R_{I}$, to ensure that the diagram of a given framed link can be drawn so that the chosen parallel coincides with the diagrammatic parallel, and I shall assume in what follows that this has been done. (Strictly we should regard the diagrams as lying on $S^{2}$ rather than $\mathbf{R}^{2}$ for framed links, to ensure that the diagrammatic representation is completely equivalent to the definition using parallel curves.)

The Homfly polynomial $P_{L}(v, z) \in \mathbf{Z}\left[v^{ \pm 1}, z^{ \pm 1}\right]$ was developed independently by several groups shortly after the discovery of the Jones polynomial [2, 6].

It is an invariant of an oriented link, characterised by the Homfly skein relation

$$
v^{-1} P(/)-v P(\lambda)=z P()()
$$

between oriented link diagrams differing only where shown. It is invariant under all three Reidemeister moves, and so $P_{L \amalg O}=\delta P_{L}$, where $\delta=\left(v^{-1}-v\right) / z$, and $L \amalg \mathrm{O}$ consists of the diagram $L$ together with a disjoint simple closed curve. Some authors replace $v$ by $v^{-1}$ in this definition. In the form given here the exponents of $v$ are predominantly positive when the crossings of $L$ are positive. 
The Homfly polynomial provides a simultaneous generalisation of the Alexander polynomial and Jones' polynomial by

$$
P_{L}(v, z)= \begin{cases}\Delta_{K}(t), & \text { the Alexander polynomial, when } v=1, z=s-s^{-1}, t=s^{2} \\ \nabla_{K}(z), & \text { Conway's version of the Alexander polynomial, when } v=1 \\ V_{K}(t), & \text { the Jones polynomial, when } v=s^{2}=t, z=s-s^{-1}\end{cases}
$$

In this original form $P$ is normalised so that the unknot $\mathrm{O}$ has invariant 1 ; in work which relates to quantum groups it is more convenient to normalise so that the empty knot $\phi$ has invariant 1 and the unknot has invariant $\delta$. I shall adopt this convention in the present work.

We may construct close relatives of the Homfly polynomial which are invariants of an oriented diagram $D$ only up to Reidemeister moves $R_{I I}$ and $R_{I I I}$ for any scalar $c$ by setting

$$
X_{D}=c^{w(D)} P_{D}(v, z),
$$

where $w(D)$ is the writhe of the diagram $D$, defined as the sum of the signs of the crossings in $D$. Thus $w(D)=\sum \varepsilon(\kappa)$, where each crossing $\kappa$ is given a sign $\varepsilon(\kappa)= \pm 1$, defined as shown,

$$
\% \varepsilon=+1, \quad \ \varepsilon=-1 .
$$

Then $X$ can be recognised by the properties

$$
X(\curlywedge)=c X(\mid)
$$

and the skein relation

$$
c^{-1} v^{-1} X(\%)-c v X(\lambda)=z X()()
$$

up to normalisation. In this way we can identify any invariant of oriented diagrams up to moves $R_{I I}$ and $R_{I I I}$ which satisfies a linear relation between

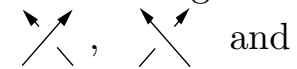
) ( with such a variant of the Homfly polynomial, provided that it multiplies by a fixed scalar $c$ under move $R_{I}$. Kauffman's bracket polynomial, for example, arises with $c=-A^{3}, z=A^{-2}-A^{2}$ and $v=A^{-4}$.

In general, when we write the relation as

$$
x^{-1} X(\%)-x X(\lambda)=z X()(),
$$

we have $X=c^{w(D)} P_{D}\left(x c^{-1}, z\right)=\left(x v^{-1}\right)^{w(D)} P_{D}(v, z)$.

Kauffman's Dubrovnik polynomial $\mathcal{D}_{L}(v, z)$, introduced in [3], is an invariant of a framed unoriented link $L$, or equivalently of unoriented link diagrams up to moves $R_{I I}$ and $R_{I I I}$. It is defined up to a constant by the linear relation

$$
\mathcal{D}(\backslash)-\mathcal{D}(\backslash)=z(\mathcal{D}()()-\mathcal{D}(\longleftarrow)),
$$


on diagrams which differ as shown, and by its behaviour

$$
\mathcal{D}(\curvearrowright)=v^{-1} \mathcal{D}(\mid)
$$

under move $R_{I}$. As above, it is appropriate here to normalise the invariant so as to take the value 1 on the empty diagram $\phi$ rather than on the unknot. The value on the unknot with framing 0 is then $1+\left(v^{-1}-v\right) / z$. It was shown by Lickorish that $\mathcal{D}$ is equivalent to Kauffman's original invariant which is defined in a similar way but with + signs in place of - signs in the linear relation.

\section{Quantum group invariants}

In this section I shall take $\mathcal{G}$ to be any of the quantum groups $\mathcal{G}_{q}$ associated to a classical Lie group $G$, with a generic choice of parameter $q$. I refer to the work of Reshetikhin and Turaev [8] for the details of the definitions of the quantum groups and associated invariants. The quantum group $\mathcal{G}$ is an algebra, in fact a Hopf algebra, over a ground ring $\Lambda$, which can frequently be taken as the Laurent polynomial $\operatorname{ring} \mathbf{Z}\left[s^{ \pm 1}\right]$, with $s^{2}=q$, or some straightforward extension of this, although it would be more correct to work with the formal power series ring $\mathbf{Q}[[h]]$ with $q=e^{h}$ and $s=e^{h / 2}$. Definition of the invariants relies crucially on the existence of an element $R$, called the 'universal $R$-matrix', in an extension of $\mathcal{G} \otimes \mathcal{G}$. The key to the construction of these invariants is the systematic representation by $\mathcal{G}$-module homomorphisms of oriented tangles whose strings have been 'coloured' by finite-dimensional $\mathcal{G}$-modules. When the components of the tangle are coloured by modules the tangle itself is represented by a homomorphism from the tensor product of the modules which colour the strings at the bottom to the tensor product of the modules which colour the strings at the top, provided that the string orientations are inwards at the bottom and outwards at the top. The dual module $V^{*}$ comes into play in place of $V$ when an arc of the tangle coloured by $V$ has an output at the bottom or an input at the top.

For example, the $(4,2)$-tangle below, when coloured as shown, is represented by a homomorphism $U \otimes W^{*} \rightarrow U \otimes X^{*} \otimes X \otimes W^{*}$.

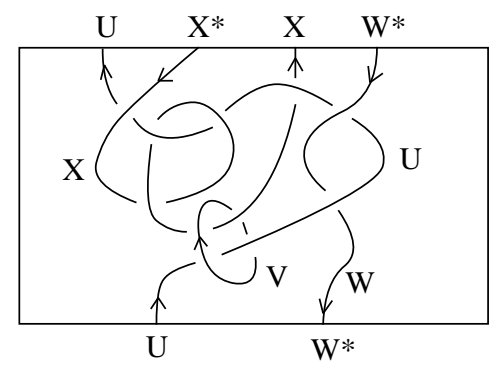

It is possible [8] to build up the definition so that the composites of consistently coloured tangles are represented by the appropriate composite homomorphisms, while one tangle alongside another is represented by the tensor product of the two corresponding homomorphisms. Every tangle can be built up as the composite of a number of 
elementary tangles which are either a simple crossing

$$
\sigma=\square \text { or } \sigma^{-1}=\square
$$

or a local maximum $\cap$ or minimum $U$, alongside a number of parallel straight strings represented by the appropriate identity homomorphism. Once it is decided how to assign a homomorphism to each of these elementary tangles, with colouring, the homomorphism for the whole tangle will be determined as a composite.

Homomorphisms for the elementary tangles are defined using the universal $R$-matrix for the tangles $\sigma^{ \pm 1}$ and the antipode in $\mathcal{G}$ for the local maximum and minimum. Two cases, depending on the orientation, must be considered for both the local maximum and the local minimum, and a little care is needed here to ensure consistency.

The final result is a definition of a homomorphism which can be shown to be invariant when the coloured tangle is altered by $R_{I I}$ and $R_{I I I}$, or deformed by isotopy. When applied to an oriented $k$-component link diagram $L$ regarded as an oriented $(0,0)$-tangle the result is a homomorphism from $\Lambda$ to $\Lambda$ for each colouring of the components of $L$ by $\mathcal{G}$-modules. This homomorphism is multiplication by an element $\mathcal{G}\left(L ; V_{1}, \ldots, V_{k}\right) \in \Lambda=$ $\mathbf{Q}[[h]]$, which is thus an invariant of the framed oriented link $L$.

This invariant $\mathcal{G}(L)$ (for a fixed quantum group $\mathcal{G}$ ) is

(1) multilinear under direct sums of modules, and

(2) multiplicative on parallels, in the sense that modules $V$ and $W$ on two parallel components in a diagram can be replaced by the single module $V \otimes W$ on a diagram with the two components amalgamated into one.

We can use (1) to extend the definition of $\mathcal{G}(L)$ to allow colouring by linear combinations of modules, and thus determine a multilinear map $\mathcal{G}(L): \mathcal{R} \times \ldots \times \mathcal{R} \rightarrow \Lambda$, where $\mathcal{R}$ is the representation ring of $\mathcal{G}$. Work of Rosso [9] shows that for generic choice of the parameter $q$ the finite dimensional representations of $\mathcal{G}$ are completely reducible, and that the representation ring $\mathcal{R}$ is isomorphic to that of the corresponding classical group.

\section{Quantum invariants which are polynomial evaluations}

I shall now consider the case of the quantum invariants when the same irreducible $V$ is used on each component of a framed oriented link $L$. By Rosso's result we may write $V \otimes V=\sum_{i=1}^{r} n_{i} V_{i}$, in $\mathcal{R}$, where each $V_{i}$ is irreducible, $V_{i} \nsucceq V_{j}$ when $i \neq j$ and $n_{i} \in \mathbf{N}$.

Theorem 1 Let $V$ be an irreducible module for the quantum group $\mathcal{G}$. Suppose that $V \otimes V \cong V_{1} \oplus V_{2}$ where $V_{1}$ and $V_{2}$ are irreducible and not isomorphic $\mathcal{G}$-modules. Then for any framed oriented link $L$ we have

$$
\mathcal{G}(L ; V, \ldots, V)=c^{w(L)} P_{L}(v, z)
$$

for some scalars $c, v$ and $z$, where $w(L)$ is the writhe of a correctly framed diagram for $L$. 
Theorem 2 Let $V$ be an irreducible module for the quantum group $\mathcal{G}$ which is isomorphic to its dual. Suppose that $V \otimes V \cong V_{1} \oplus V_{2} \oplus V_{3}$ where $V_{1}, V_{2}$ and $V_{3}$ are irreducible and not isomorphic. Then for any framed link $L$ we have

$$
\mathcal{G}(L ; V, \ldots, V)=\mathcal{D}_{L}(v, z)
$$

for some $v$ and $z$.

Remark. In this case exactly one of the summands, $V_{3}$ say, must be the trivial module.

Proof of theorem 1: $\quad$ Every $\mathcal{G}$-module endomorphism of $V \otimes V$ must preserve the two irreducible summands $V_{1}$ and $V_{2}$, and will act on each by scalar multiplication, by scalars $a_{1}$ and $a_{2}$ respectively. These scalars determine the endomorphism, and so any three endomorphisms of $V \otimes V$ must be linearly dependent. Now the three tangles

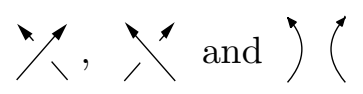

when coloured throughout by $V$ are represented by three endomorphisms $R, R^{-1}$ and Id of $V \otimes V$. These must satisfy some linear relation, which can be written as

$$
x^{-1} R-x R^{-1}=z \mathrm{Id}
$$

for some scalars $x$ and $z$. Suppose now that we have diagrams of three framed links $D_{ \pm}$and $D_{0}$, differing only by the three tangles shown above. We may use the diagrams to calculate the invariant $\mathcal{G}(L ; V \ldots, V)$ for each link, by composing module homomorphisms. The homomorphisms given by the three diagrams will be identical, except that at some level the homomorphism $R$ used in $D_{+}$is replaced either by $R^{-1}$ for $D_{-}$or Id for $D_{0}$. The composite homomorphisms, and thus the eventual invariants, must then satisfy the same linear relation, giving

$$
x^{-1} \mathcal{G}\left(D_{+} ; V, \ldots, V\right)-x \mathcal{G}\left(D_{-} ; V, \ldots, V\right)=z \mathcal{G}\left(D_{0} ; V, \ldots, V\right) .
$$

Furthermore, since $V$ is irreducible, the $(1,1)$-tangle $\bigcirc$ when coloured by $V$ must be represented by some scalar multiple, $c$ say, of the identity on $V$, by Schur's lemma. When $\bigcirc$ is replaced by $/$ in a diagram the invariant $\mathcal{G}(D ; V, \ldots, V)$ is then multiplied by $c$. It follows from the properties of the Homfly polynomial discussed above that $\mathcal{G}(D ; V, \ldots, V)$ can be identified with a variant $X_{D}$ of the Homfly polynomial. Since $\mathcal{G}(D)$ has the value 1 on the empty diagram the exact formula is

$$
\mathcal{G}(D ; V, \ldots, V)=c^{w(D)} P_{D}\left(x c^{-1}, z\right),
$$

where $x, c$ and $z$ have the values determined by the relations.

Remark. The endomorphism $R$ satisfies the quadratic relation $R^{2}-x z R-x^{2}=0$; if we set $z=s-s^{-1}$ then the eigenvalues of $R$ are $x s$ and $-x s^{-1}$ and its eigenspaces are the two submodules $V_{1}$ and $V_{2}$ making up the decomposition of $V \otimes V$. 
Proof of theorem 2: With three irreducible summands for $V \otimes V$ each endomorphism is determined by three scalars, and so any four endomorphisms of $V \otimes V$ will be linearly dependent.

Because $V$ is isomorphic to its dual $V^{*}$ the tangle $\square$, when its components are coloured by the module $V$, will be represented by an endomorphism, $H$ say, of $V \otimes V$. We then have a linear relation between the four endomorphisms $R^{ \pm 1}$, Id and $H$, where $R^{ \pm 1}$ represent the tangles $\sigma^{ \pm 1}$ when coloured by $V$. Suppose then that

$$
a R+b R^{-1}=c \mathrm{Id}+d H
$$

for some scalars $a, b, c$ and $d$.

Write $V(L)$ for the invariant $\mathcal{G}(L ; V, \ldots, V)$ of the framed link $L$. The linear relation between the endomorphisms gives a similar relation as before among the invariants where the diagrams only differ as shown, thus

$$
a V(\)+b V(\backslash)=c V()()+d V(\longleftarrow) .
$$

Again the irreducibility of $V$ shows that there is some scalar $v$ with $V(\curvearrowright)=v^{-1} V(\mid)$. We can then identify $V(L)$ with the Dubrovnik invariant of $L$ in one of its forms if we can show that either $a+b=c+d=0$ or that $a=b$ and $c=d$.

We may redraw the tangle $\sigma^{-1}$ by isotopy as another composite of elementary tangles in the way shown.

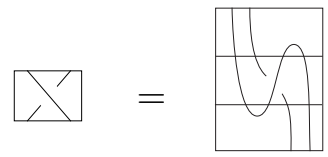

By the theory of quantum group invariants, the endomorphism $R^{-1}$ can equally be expressed as the appropriate composite of three homomorphisms. Now one of the factors in the composite involves the tangle $\sigma$ which is represented by $R$, and this can be replaced, using the linear relation, by a combination of $R^{-1}, H$ and Id. This gives a linear relation between the composite endomorphisms arising from four tangles, as indicated.

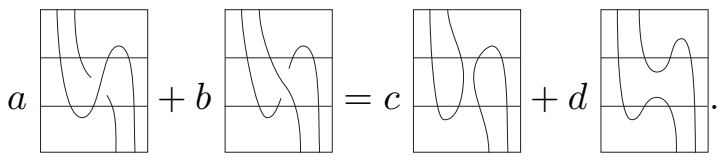

Since these tangles be simplified by isotopy the endomorphisms which represent them are just $R^{ \pm 1}, H$ and Id, giving the equation

$$
a R^{-1}+b R=c H+d \mathrm{Id} .
$$

Subtracting the two equations gives

$$
R-R^{-1}=z(\operatorname{Id}-H), \text { with } z=\frac{c-d}{a-b},
$$

unless $a=b$. Then $V(L)=\mathcal{D}(v, z)$. 
If $a=b$ then $(c-d)(\mathrm{Id}-H)=0$ and so $c=d$ since $H \neq \mathrm{Id}$. This gives

$$
R+R^{-1}=z(\operatorname{Id}+H), \text { with } z=c / a,
$$

and hence $V(L)$ is the other version of the Dubrovnik invariant.

Remark. The endomorphism $R$ has three eigenspaces, $V_{1}, V_{2}$ and $V_{3}$, with eigenvalues $a_{1}, a_{2}$ and $a_{3}$ say. The endomorphism $H$ represents the composite of a $(2,0)$-tangle with a $(0,2)$-tangle, and hence factors through the trivial module. It must then be a scalar multiple of the projection to the trivial summand of $V \otimes V$, which we can take to be $V_{3}$. It follows that $R H=a_{3} H$. We can draw a tangle represented by $H R$ which differs from the tangle represented by $H$ simply by a left-hand curl, and so $H R=v H$, so that $a_{3}=v$.

Now $-z H=R-R^{-1}-z$ using the standard Dubrovnik signs and so

$$
\left(R-R^{-1}-z\right)(R-v)=0 .
$$

The other two eigenvalues of $R$ must then be the roots $s,-s^{-1}$ of the equation $R^{2}-$ $z R-1=0$ where $z=s-s^{-1}$.

Applications. The structure of the representation ring for the generic quantum group is the same as that of the corresponding classical Lie group. When $G=S l(k)$ or $S U(k)$ the fundamental $k$-dimensional representation $V$ has the property required in theorem 1. The representation of $G$ on $V \otimes V$ can be viewed as the representation of $G$ on the space $\mathcal{M}$ of $k \times k$ matrices using the map $G \times \mathcal{M} \rightarrow \mathcal{M}$ defined by $(P, A) \mapsto P^{T} A P$. This decomposes into two irreducible representations, on the symmetric and skew-symmetric matrices respectively, of dimension $k(k+1) / 2$ and $k(k-1) / 2$.

There exists a corresponding $k$-dimensional representation $V_{\square}$ of the quantum group $\mathcal{G}=S U(k)_{q}$ whose product $V_{\square} \otimes V_{\square}$ decomposes as the sum of two irreducible representations of dimensions $k(k+1) / 2$ and $k(k-1) / 2$. Theorem 1 then yields Turaev's well-known result [10] that the invariant $V_{\square}(L)$ is given by $P_{L}(v, z)$, after appropriate normalisation, for some choice of $v$ and $z$. The endomorphism $R$ of $V_{\square} \otimes V_{\square}$ may be altered by a scalar multiple $x$ to yield one of the variants $X$ for the Homfly polynomial; this only changes the invariant by a factor depending on the writhe of the diagram, without changing the values of $v$ and $z$. Turaev uses a multiple $R$ of the universal $R$-matrix which satisfies the equation (*) with $x=1$, and has $z=s-s^{-1}, v=s^{-k}$ in our notation. When working with the endomorphism given by Drinfeld's universal $R$-matrix [1] the parameter $x$ has value $x=s^{-1 / k}=e^{-h / 2 k}$. In the applications given here it is important to use the universal $R$-matrix, to allow accurate calculation of the invariants on parallels and satellites.

In what follows, for $\mathcal{G}=S U(k)_{q}$, I shall then use the endomorphism $R$ for $V_{\square} \otimes V_{\square}$ which satisfies $(*)$ with $x=s^{-1 / k}, z=s-s^{-1}$. This endomorphism has eigenvalues $x s$ and $-x s^{-1}$. The corresponding eigenspaces are the irreducible submodules of dimension $k(k+1) / 2$ and $k(k-1) / 2$ respectively, since $R$ reduces to the simple interchange of factors when we put $h=0$ and thus $x=s=1$ in the quantum group; the symmetric summand of dimension $k(k+1) / 2$ in the classical case is the one with eigenvalue 1 . 
The tensor product $V \otimes V$ of the fundamental $k$-dimensional representation of $S O(k)$ decomposes similarly into three irreducibles corresponding to scalar matrices, symmetric matrices of trace 0 and skew-symmetric matrices. Theorem 2 then confirms the direct observation of Turaev; in this case again $z=s-s^{-1}$ while $v=s^{-(k-1)}$.

\section{Two special cases}

The conditions of theorem 2 are satisfied also in two special cases besides the fundamental representations of $S O(k)$. These are both suggested by coincidences in the theory of Lie algebras, where $S U(2)$ and $S O(3)$ have the same Lie algebra, as do $S U(4)$ and $S O(6)$. The 3-dimensional irreducible representation of $S U(2)$, and equally the 6-dimensional irreducible representation of $S U(4)$ in the quantum group setting might then be expected to be the fundamental representations of $S O(3)$ and $S O(6)$, and thus determine the corresponding specialisations of the Dubrovnik invariant. Here we use theorem 2, without attempting to make an explicit identification of the pairs of quantum groups, to show that this is indeed the case.

The representations in question for $S U(2)$ and $S U(4)$ correspond to one of the two summands of the 2-fold tensor product of the fundamental representation, and thus classically to either symmetric $2 \times 2$ matrices or skew-symmetric $4 \times 4$ matrices. To apply theorem 2 it is enough to show that the 2-fold tensor product of each of these representations decomposes as the sum of three irreducibles, one of which is the trivial representation.

Let $V$ be one of these two summands in the case of the general unitary quantum group $\mathcal{G}=S U(k)_{q}$. The representation ring of $\mathcal{G}$ is isomorphic to that of $S U(k)$ itself, so we can use classical information to determine how tensor products of $\mathcal{G}$-modules decompose. The irreducible representations of $S U(k)$ are described classically by Young diagrams, which are suitably arranged arrays of cells. The fundamental representation corresponds to the diagram $\square$, while the symmetric and skew-symmetric summands of its 2 -fold tensor product correspond to $\square$ and $\boxminus$ respectively. We need to know the decomposition of the tensor products $\square \otimes \square$ and $\boxminus \otimes 日$ into irreducible summands. Classical procedures show that each can be decomposed as the sum of three irreducibles, with Young diagrams as shown

$$
\begin{gathered}
\square \otimes \square=\square+\boxplus+\boxplus \\
\boxminus \otimes 日=\text { 日 }+ \text { 田 }+\boxplus .
\end{gathered}
$$

For a given $k$, not all Young diagrams correspond to distinct irreducibles. Any diagram with more than $k$ rows of cells gives the zero representation, the diagram with 1 column and $k$ cells gives the trivial (scalar) representation, while any column with $k$ cells can be removed from a diagram without altering the corresponding irreducible.

We can then see that for $k=2$ the product $\square \otimes \square$ contains one trivial summand $\boxplus$, while $\boxminus \otimes 日$ contains the trivial summand 目 in the case $k=4$. The conditions 
for theorem 2 are then satisfied in each case, and the corresponding quantum invariants are each specialisations of the Dubrovnik invariant.

We can be more specific and identify the values of the parameters $v$ and $z$ in terms of $s$ by the following theorem.

Theorem 3 The invariant $V(L)=\mathcal{G}(L ; V, \ldots, V)$ satisfies

$$
V(L)=\mathcal{D}_{L}\left(s^{-4}, s^{2}-s^{-2}\right)
$$

when $\mathcal{G}=S U(2)_{q}$ and $V=V_{\square}$ is the 3-dimensional irreducible,

$$
V(L)=\mathcal{D}_{L}\left(s^{-5}, s-s^{-1}\right)
$$

when $\mathcal{G}=S U(4)_{q}$ and $V=V_{\text {日 }}$ is the 6-dimensional irreducible.

Proof: As noted above, theorem 2 applies to show that $V(L)=\mathcal{D}_{L}(v, z)$ for some $v$ and $z$ in each of these cases. To identify the parameters $v$ and $z$ it is enough to find the three eigenvalues of the endomorphism $R$ of $V \otimes V$. By the remark following theorem 2 the eigenvalue associated with the trivial representation will be $v$, while the sum of the other two, whose product is -1 , will give $z$.

It will be enough to identify the eigenvalues up to sign; this will determine $v$ and $z$ up to sign. We may then confirm the theorem by checking in the case when $L$ is a simple closed curve.

As part of the proof I shall now discuss some features of the invariants when $\mathcal{G}$ is any of the unitary quantum groups $S U(k)_{q}$, using further properties of the classical representation theory of $S U(k)$. As mentioned in the previous section, irreducible $\mathcal{G}$-modules (representations of $\mathcal{G}$ ) are indexed by Young diagrams. Write $V_{\lambda}$ for the irreducible $\mathcal{G}$ module with Young diagram $\lambda$. Then the fundamental module is $V_{\square}$ and its symmetric square and exterior square are respectively $V_{\square}$ and $V_{\boxminus}$. The tensor product $\left(V_{\square}\right)^{\otimes n}$ of $n$ copies of $V_{\square}$ decomposes as a direct sum of modules $\sum W_{\lambda} \otimes V_{\lambda}$, where the sum is taken over all admissible Young diagrams with $n$ cells, and each $W_{\lambda}$ is a trivial $\mathcal{G}$-module of dimension $d_{\lambda}$. This dimension $d_{\lambda}$ is independent of $k$ and can be determined classically. It is given inductively by $d_{\lambda}=\sum d_{\lambda^{\prime}}$, where $\lambda^{\prime}$ runs over Young diagrams derived from $\lambda$ by removing one cell.

Representation of coloured tangles, and thus coloured braids, by $\mathcal{G}$-module endomorphisms determines a representation of the $n$-string braid group $B_{n}$ by $\mathcal{G}$-module endomorphisms of $\left(V_{\square}\right)^{\otimes n}$. Each elementary braid $\sigma_{i}$ is represented by the tensor product of suitable identity endomorphisms and the endomorphism $R: V_{\square} \otimes V_{\square} \rightarrow V_{\square} \otimes V_{\square}$, whose eigenvalues have the form $x s$ and $-x s^{-1}$, with $x=s^{-1 / k}$ when dealing with $S U(k)_{q}$, as noted above. The corresponding eigenspaces are $V_{\square}$ and $V_{\boxminus}$ respectively.

Write $|\lambda|$ for the number of cells in the Young diagram $\lambda$. The representation of $B_{n}$ determines a representation of $B_{n}$ on each space $W_{\lambda}$ with $|\lambda|=n$ since the summands $W_{\lambda} \otimes V_{\lambda}$ in the tensor product must be preserved by the module endomorphisms. Each elementary braid $\sigma_{i}$ is then represented on $W_{\lambda}$ with eigenvalues $x s$ and $-x s^{-1}$. Write 
$a_{\lambda}$ for the number of eigenvalues $x s$ and $b_{\lambda}\left(=d_{\lambda}-a_{\lambda}\right)$ for the number of eigenvalues $-x s^{-1}$; these numbers are independent of $i$ since all the braids $\sigma_{i}$ are conjugate in $B_{n}$. Set $\sigma_{\lambda}=a_{\lambda}-b_{\lambda}$, the signature of the representing matrix when $x=s=1$. When we put $h=0$ and thus $x=s=1$ the representation of $B_{n}$ becomes a representation of the symmetric group $S_{n}$, and then $\sigma_{\lambda}$ is the signature of any matrix representing a transposition. The signatures $\sigma_{\lambda}$ can be determined classically. Since a transposition acting on $V_{\square} \otimes V_{\square}$ classically preserves the symmetric tensors, and acts with eigenvalue -1 on the skew symmetric tensors we will have $a_{\lambda}=1$ and $b_{\lambda}=0$ when $\lambda=\square$, corresponding to the symmetric case, while $a_{\lambda}=0$ and $b_{\lambda}=1$ when $\lambda=\boxminus$. The signature satisfies an inductive relation as for $d_{\lambda}$, namely that $\sigma_{\lambda}=\sum \sigma_{\lambda^{\prime}}$ where $\lambda^{\prime}$ again runs over Young diagrams given from $\lambda$ by removing one cell. The induction starts with $\sigma_{\square}=1$ and $\sigma_{日}=-1$. The endomorphism of $W_{\lambda}$ representing each $\sigma_{i}$ then has determinant $x^{d_{\lambda}} s^{\sigma_{\lambda}}(-1)^{b_{\lambda}}$, and hence the full-twist braid $\Delta_{n}^{2}$ acting on $W_{\lambda}$ has determinant $\left(x^{d_{\lambda}} s^{\sigma_{\lambda}}\right)^{n(n-1)}$.

The simple curl $\bigcirc$ when coloured with the irreducible module $V_{\lambda}$ is represented by some multiple, $c_{\lambda}$ say, of the identity on $V_{\lambda}$. The endomorphism of $\left(V_{\square}\right)^{\otimes n}$ which represents the tangle

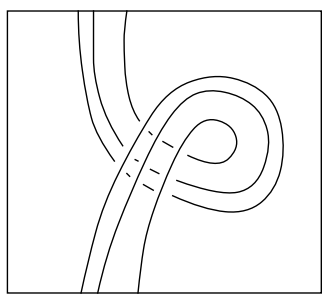

when coloured by $V_{\square}$ restricts to an endomorphism of the summand $W_{\lambda} \otimes V_{\lambda}$ which may be seen, using the behaviour of quantum invariants on parallels, to be scalar multiplication by $c_{\lambda}$. On the other hand, this tangle can be altered by removing one curl from each string to become the braid $\Delta_{n}^{2}$ and it is thus represented as an endomorphism of $\left(V_{\square}\right)^{\otimes n}$ by $\left(c_{\square}\right)^{n} \Delta_{n}^{2}$. Calculating the determinant of this endomorphism on $W_{\lambda}$ in two different ways gives

$$
\left(c_{\lambda}\right)^{d_{\lambda}}=\left(c_{\square}\right)^{n d_{\lambda}}\left(x^{d_{\lambda}} s^{\sigma_{\lambda}}\right)^{n(n-1)} .
$$

Now $c_{\square}=x v^{-1}$ with $v=s^{-k}, x=s^{-1 / k}$ for $S U(k)_{q}$ and we may then take $d_{\lambda}$-th roots to write (up to a root of unity)

$$
c_{\lambda}=x^{|\lambda|^{2}} v^{-|\lambda|} s^{\sigma_{\lambda}|\lambda|(|\lambda|-1) / d_{\lambda}} .
$$

We can now construct the following table for $d, \sigma$ and $c$ up to a $d$ th root of unity, starting from the data that $d_{\square}=1$ and $\sigma_{\square}=1$, while $d_{\boxminus}=1$ and $\sigma_{\boxminus}=-1$. 


$$
\begin{array}{ccrc}
\lambda & d & \sigma & c \\
\square & 1 & 1 & x^{4} v^{-2} s^{2} \\
\text { 曰 } & 1 & -1 & x^{4} v^{-2} s^{-2} \\
\boxplus & 2 & 0 & x^{16} v^{-4} \\
\boxplus & 3 & 1 & x^{16} v^{-4} s^{4} \\
\text { 曰 } & 3 & -1 & x^{16} v^{-4} s^{-4} \\
\text { 口 } & 1 & 1 & x^{16} v^{-4} s^{12} \\
\text { 目 } & 1 & -1 & x^{16} v^{-4} s^{-12} .
\end{array}
$$

Write $R_{\mu \mu}: V_{\mu} \otimes V_{\mu} \rightarrow V_{\mu} \otimes V_{\mu}$ for the endomorphism representing the elementary tangle $/$ when coloured with $V_{\mu}$. A similar argument shows that $R_{\mu \mu}^{2}$, representing the 2-braid $\sigma_{1}^{2}=\Delta_{2}^{2}$, acts by scalar multiplication on any of the irreducible summands $V_{\nu}$ of $V_{\mu} \otimes V_{\mu}$, and that the scalar is $c_{\mu}^{-2} c_{\nu}$. The endomorphism $R_{\mu \mu}$ of $V_{\mu} \otimes V_{\mu}$ then has eigenvalues, up to sign, of $c_{\mu}^{-1} \sqrt{c_{\nu}}$.

To identify the parameters $v$ and $z$ in cases (1) and (2) of the theorem we must find the eigenvalues of $R_{\mu \mu}$ when $\mu=\square, \mathcal{G}=S U(2)_{q}$ for (1) and when $\mu=\boxminus, \mathcal{G}=S U(4)_{q}$ for (2). We know in each case that $v=c_{\mu}^{-1}$ will be one eigenvalue, and that the sum of the other two eigenvalues, whose product is -1 , will give us the value of $z$.

We can find the three eigenvalues, up to sign, as $c_{\mu}^{-1} \sqrt{c_{\nu}}$ where $\nu$ runs through the three summands of $\mu \otimes \mu$. For $\mu=\square$ the table above gives eigenvalues, up to sign, of $x^{4} s^{-2}, x^{4}$ and $x^{4} s^{4}$, which have values $s^{-4}, s^{-2}$ and $s^{2}$ for $S U(2)_{q}$. In this case $v=c_{\mu}^{-1}=s^{-4}$ and so $z= \pm\left(s^{2}-s^{-2}\right)$. Similarly, when $\mu=$ 日, the eigenvalues, again up to sign, are $x^{4} s^{2}, x^{4}$ and $x^{4} s^{-4}$, which have the values $s, s^{-1}$ and $s^{-5}$ for $S U(4)_{q}$. Here we can calculate $c_{\mu}^{-1}=s^{-5}$ from the table, and so $v=s^{-5}$, while $z= \pm\left(s-s^{-1}\right)$.

It then only remains to identify the choice of sign for $z$ in each case, which we do by considering the invariant $V_{\mu}(U)$, where $U$ is the unknot with framing 0 . We could use the results from [4], as in the next theorem, to calculate $V_{\mu}(U)$ and compare it with $\mathcal{D}_{U}(v, z)$.

A quicker check however can be made as follows. We know that the value of a general quantum group invariant $\mathcal{G}\left(L ; V_{\mu}\right)$ for a knot $L$ when we put $h=0$ is the dimension of the module $V_{\mu}$. Now $\mathcal{D}_{U}(v, z)=1+\left(v^{-1}-v\right) / z$. In case (1) we have $v=s^{-4}, z= \pm\left(s^{2}-s^{-2}\right)$ and so $\mathcal{D}_{U}(v, z)=1 \pm\left(s^{2}-s^{-2}\right)$, while $\operatorname{dim} V_{\square}=3$ for $S U(2)$. We must therefore take $z=s^{2}-s^{-2}$. In case (2) we have $v=s^{-5}, z= \pm\left(s-s^{-1}\right)$ and then

$$
\mathcal{D}_{U}(v, z)=1 \pm \frac{s^{5}-s^{-5}}{s-s^{-1}}=1 \pm\left(s^{4}+s^{2}+1+s^{-2}+s^{-4}\right)
$$

while $\operatorname{dim} V_{\boxminus}=6$ for $S U(4)$. Thus we must take $z=s-s^{-1}$. This completes the check so the theorem follows.

Remark. It is established [7, 11] that the invariants $V(L)$ for the unitary quantum groups can be calculated in terms of Homfly polynomials of suitable satellites of $L$. An explicit account in [4] relates the cases $V=V_{\square}$ and $V=V_{\text {日 }}$ to the Homfly polynomials 
of simple 2-cables about $L$, with the variables $v$ and $z$ replaced, for $S U(k)$, by $s^{-k}$ and $s-s^{-1}$ respectively. Consequently there is a relation between these Homfly polynomials and the specialisations of the Dubrovnik polynomial in theorem 3.

In the first case this yields Yamada's result [12] relating the Jones polynomial of the 2-parallel of $L$ to the specialisation of the Dubrovnik polynomial $\mathcal{D}_{L}\left(s^{4}, s^{-2}-s^{2}\right)$ where $s^{2}=t$ for the parameter in the Jones polynomial.

The second case gives a new relation, which I state here for the case of an oriented framed knot $C$, but which extends naturally to the case of a link.

Theorem 4 Let $C$ be a framed knot. Then

$$
\mathcal{D}_{C}\left(s^{-5}, s-s^{-1}\right)=s^{15 w(C)} \frac{1}{1+s^{-2}}\left(P_{C(2,0)}\left(s^{-4}, s-s^{-1}\right)-s^{3} P_{C(2,1)}\left(s^{-4}, s-s^{-1}\right)\right),
$$

where $w(C)$ is the writhe of a correctly framed diagram of $C$, and $C(2,0), C(2,1)$ denote the $(2,0)$ and $(2,1)$ cables about $C$ relative to the chosen framing of $C$.

Proof: The quantum invariant $\mathcal{G}\left(C ; V_{\lambda}\right)$ for a unitary irreducible module $V_{\lambda}$ is related explicitly in [[4]] to the Homfly polynomials of cables with $|\lambda|$ strings about $C$. From a braid $\beta$ on $n$ strings we may construct a satellite $C(\beta)$ of $C$ by taking $n$ parallel strings which follow the framing around the diagram of $C$ and inserting the braid $\beta$ as indicated.

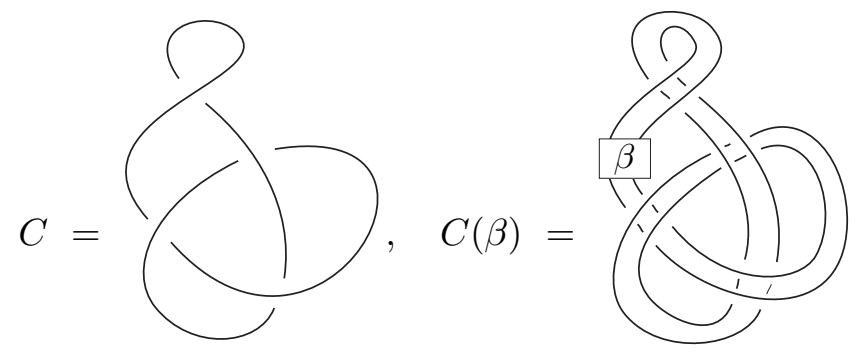

When $n=2$ and $\beta=\sigma_{1}$ this satellite is called the $(2,1)$-cable about the framed knot $C$; with $\beta=1$, the identity braid, we get the $(2,0)$-cable consisting of two parallel strands about $C$.

Write $P_{C}(\hat{\beta})$ for the Homfly polynomial of $C(\beta)$, and extend this notation to linear combinations of braids $\beta=\sum a_{i} \beta_{i}$ in place of $\beta$ to mean the linear combination of Homfly polynomials, so that $P_{C}(\hat{\beta})=\sum a_{i} P_{C}\left(\hat{\beta}_{i}\right)$. For $\mathcal{G}=S U(k)_{q}$ it is shown in [4] that

$$
\mathcal{G}\left(C ; V_{\text {日 }}\right)=\left(x v^{-1}\right)^{4 w(C)} P_{C}\left(\hat{e}_{日}\right),
$$

where $e_{\mathrm{E}}=\left(1-s^{-1} v^{-1} \sigma_{1}\right) /\left(1+s^{-2}\right)$, and the substitutions $v=s^{-k}, z=s-s^{-1}$ and $x=s^{-1 / k}$ are made on the right-hand side.

Here we have $k=4$ and so we get

$$
\mathcal{G}\left(C ; V_{\boxminus}\right)=s^{15 w(C)} \frac{1}{1+s^{-2}}\left(P_{C}(\hat{1})-s^{3} P_{C}\left(\hat{\sigma}_{1}\right)\right) .
$$


The result of theorem 3(2) allows the left-hand side to be replaced by the specialisation of the Dubrovnik polynomial as claimed.

Remark. The detailed formulae in theorems 3 and 4 have been amended from an earlier version of the paper in which substitutions $v=s^{k}$ and $x=s^{1 / k}$ were used. These did not tally exactly with the results of Turaev and Drinfeld, and were more suited to the choice $s=-1$ rather than $s=1$ when making the specialisation $q=1$.

\section{References}

[1] Drinfeld, V.G. Quantum groups, in Proceedings of the International Congress of Mathematicians, Berkeley 1986, (Amer. Math. Society 1987), 798-820.

[2] Freyd, P., Yetter, D., Hoste, J., Lickorish, W.B.R., Millett, K.C. and Ocneanu, A. A new polynomial invariant of knots and links, Bull. Amer. Math. Soc. 12 (1985), 239-246.

[3] Kauffman, L.H. An invariant of regular isotopy, Trans. Amer. Math. Soc. 318 (1990), 417-471.

[4] Morton, H.R. Invariants of links and 3-manifolds from skein theory and from quantum groups, to appear in 'Topics in knot theory', the Proceedings of the NATO Summer Institute in Erzurum 1992, Kluwer.

[5] Morton, H.R. and Strickland, P.M. Jones polynomial invariants for knots and satellites, Math. Proc. Cambridge Philos. Soc. 109 (1991), 83-103.

[6] Przytycki, J. and Traczyk, P. Invariants of links of Conway type, Kobe J. Math. 4 (1987), 115-139.

[7] Reshetikhin, N. Y. Quantized universal enveloping algebras, the Yang-Baxter equation and invariants of links I and II , preprint, LOMI E-4-87, 1987.

[8] Reshetikhin, N. Y. and Turaev, V. G. Ribbon graphs and their invariants derived from quantum groups, Comm. Math. Phys. 127 (1990), 1-26.

[9] Rosso, M. Finite dimensional representations of the quantum analog of the enveloping algebra of a complex simple Lie algebra, Comm. Math. Phys. 117 (1988), 581-593.

[10] Turaev, V.G. The Yang-Baxter equation and invariants of links, Invent. Math. 92 (1988), 527-553.

[11] Wenzl, H. Representations of braid groups and the quantum Yang-Baxter equation, Pacific J. Math. 145 (1990), 153-180.

[12] Yamada, S. An operator on regular isotopy invariants of link diagrams, Topology 28 (1989), 369-377. 